\title{
Comment on 'Transarterial chemo-embolisation of hepatocellular carcinoma: impact of liver function and vascular invasion'
}

\author{
Teh-la Huo* ${ }^{\star 1,2,3}$, Po-Hong Liu ${ }^{2,4}$ and Chia-Yang Hsu ${ }^{2,5}$
}

${ }^{1}$ Department of Medicine, Taipei Veterans General Hospital, Taipei, Taiwan; ${ }^{2}$ Faculty of Medicine, National Yang-Ming University School of Medicine, Taipei, Taiwan; ${ }^{3}$ Institute of Pharmacology, National Yang-Ming University School of Medicine, Taipei, Taiwan; ${ }^{4}$ Harvard T.H. Chan School of Public Health, Boston, MA, USA and ${ }^{5}$ Department of Internal Medicine, University of Nevada School of Medicine, Reno, NV, USA

Sir,

We read the paper 'Transarterial chemo-embolisation of hepatocellular carcinoma: impact of liver function and vascular invasion' with interest, which was published in a recent issue of British Journal of Cancer (Waked et al, 2017). In this elegant, multi-center study, including 3030 patients with hepatocellular carcinoma (HCC) undergoing transarterial chemo-embolisation, authors demonstrate that the ALBI grade can be used as a marker of liver functional reserve in HCC patients from different geographical regions. We have consistent findings from our own recent study, which enrolled a large cohort of 3182 HCC patients (Liu et al, 2017). In addition, an independent study from Japan confirmed the predictive role of ALBI grade in 2584 HCC patients (Hiraoka et al, 2016). All these studies meticulously show the feasibility of the ALBI grade as a prognostic predictor for HCC. Although the findings are interesting, some concerns may deserve our attention.

The major prognostic predictors of HCC include liver dysfunction, tumour extent, general health status and treatment modality. The BCLC staging system incorporates these key factors and includes treatment suggestions for different stages. Although the BCLC system has been integrated in the current management guidelines, a recently proposed HKLC staging system offered better prognostic ability compared with BCLC system (Yau et al, 2014). Besides the BCLC and HKLC systems, multiple staging systems were proposed and generally claimed to have superior prognostic performance. This lack of consensus may stem from heterogeneity of liver pathology and diverse treatment modalities, which complicate the pursuit for an ideal staging model (Liu et al, 2016). Therefore, the ALBI grade, as a potentially better biomarker of liver functional reserve and survival predictor, should be tested in the existing HCC staging models to investigate if it can replace the traditional markers, including Child-Turcotte-Pugh (CTP) and model for end-stage liver disease (MELD) score.

To further improve the performance of the ALBI grade, a more recent proposal showed that adding platelet count into the ALBI equation, or the PALBI grade, could enhance the prognostic ability of the ALBI grade (Roayaie et al, 2015). PALBI score was calculated as the following equation: $\quad 2.02 \times \log _{10}$ bilirubin-0.37 $\times\left(\log _{10} \quad \text { bilirubin }\right)^{2}-0.04 \times$ albumin-3.48 $\times \log _{10}$ platelets $+1.01 \times\left(\log _{10} \text { platelets }\right)^{2}$. PALBI grade was defined as grade 1 (score $\leqslant-2.53$ ), grade 2 (score $>-2.53$ and $\leqslant-2.09$ ), and grade 3 (score $>-2.09$ ). The feasibility of this 'updated' version of the ALBI grade in predicting the survival for HCC patients has been validated in our patient cohort (Liu et al, 2017). In this study, we show that the PALBI score may outperform the ALBI grade based on several reasons. First, PALBI grade incorporates blood platelet count as an indicator for the severity of portal hypertension. Second, many HCC patients are CTP class A, and the PALBI grade can further differentiate into three distinct prognostic groups. As such, the PALBI grade could be a more statistically robust model that provides higher discriminatory power for HCC.

In summary, a more in-depth evaluation of the intrinsic nature of the ALBI and PALBI grade is required to consolidate their application in clinical practice. Undoubtedly, it is crucially important to implement ALBI/PALBI-based models into current HCC staging systems to investigate if the prognostic ability can be further enhanced. Further studies from international cohorts are needed to address these issues.

\section{AUTHOR CONTRIBUTIONS}

Guarantor of the article: T-IH. Specific author contributions: T-IH and $\mathrm{P}-\mathrm{HL}$ performed the research. C-YH, P-HL and T-IH designed the study and wrote the paper. All authors approved the final version of the manuscript.

\section{CONFLICT OF INTEREST}

The authors declare no conflict of interest.

\section{REFERENCES}

Hiraoka A, Kumada T, Michitaka K, Toyoda H, Tada T, Ueki H, Kaneto M, Aibiki T, Okudaira T, Kawakami T, Kawamura T, Yamago H, Suga Y, Miyamoto Y, Tomida H, Azemoto N, Mori K, Miyata H, Ninomiya T, Kawasaki H (2016) Usefulness of albumin-bilirubin grade for evaluation of prognosis of 2584 Japanese patients with hepatocellular carcinoma. $J$ Gastroenterol Hepatol 31: 1031-1036.

Liu PH, Hsu CY, Hsia CY, Lee YH, Chiou YY, Huang YH, Lee FY, Lin HC, Hou MC, Huo TI (2017) ALBI and PALBI grade predict survival for HCC across treatment modalities and BCLC stages in the MELD era. J Gastroenterol Hepatol 32: 879-886.

Liu PH, Hsu CY, Hsia CY, Lee YH, Su CW, Huang YH, Lee FY, Lin HC, Huo TI (2016) Prognosis of hepatocellular carcinoma: assessment of eleven staging systems. J Hepatol 64: 601-608.

Roayaie S, Jibara G, Berhane S (2015) PALBI- an Objective Score Based on Platelets, Albumin and Bilirubin Stratifies HCC Patients Undergoing Resection and Ablation Better Than Child's Classification. The Liver Meeting. American Association for the Study of Liver Diseases: San Francisco, CA, USA.

Waked I, Berhane S, Toyoda H, Chan SL, Stern N, Palmer D, Tada T, Yeo W, Mo F, Bettinger D, Kirstein MM, Iñarrairaegui M, Gomaa A, Vogel A, Meyer T, Sangro B, Lai P, Kumada T, Johnson PJ (2017) Transarterial chemoembolisation of hepatocellular carcinoma: impact of liver function and vascular invasion. Br J Cancer 116: 448-454.

Yau T, Tang VY, Yao TJ, Fan ST, Lo CM, Poon RT (2014) Development of Hong Kong Liver Cancer staging system with treatment stratification for patients with hepatocellular carcinoma. Gastroenterology 146: 1691-1700.

This work is published under the BJC's standard license to publish agreement After 12 months the license terms will change to a Creative Commons AttributionNonCommercial-Share Alike 4.0 Unported License. 